\title{
A CHARACTERIZATION OF THE 2-SPHERE BY EIGENFUNCTIONS
}

\author{
SHIU-YUEN CHENG
}

\begin{abstract}
The spheres in 3-dim Euclidean space are characterized by the fact that they have three 1st eigenfunctions with square sum equal to a constant.
\end{abstract}

0 . Introduction. Suppose that $(M, g)$ is an $n$-dim $C^{\infty}$ compact Riemannian manifold without boundary. The Laplacian operator acting on functions, denoted by $\Delta$, is defined by

$$
\sum_{i, j} \frac{1}{\sqrt{g}} \frac{\partial}{\partial x_{i}} \sqrt{g} g^{i j} \frac{\partial}{\partial x_{j}},
$$

where $\left(x_{1}, \ldots, x_{n}\right)$ is a local coordinate system, $g_{i j} d x_{i} d x_{j}$ is the fundamental tensor, $\left(g^{i j}\right)=\left(g_{i j}\right)^{-1}$ and $g=\operatorname{det}\left(g_{i j}\right)$. It is well known that the eigenvalue problem $\Delta \varphi+\mu \varphi=0$ has discrete eigenvalues, we list them as

$$
0=\mu_{0}(M)<\mu_{1}(M) \leqslant \mu_{2}(M) \leqslant \mu_{3}(M) \leqslant \ldots .
$$

We call $\mu_{i}(M)$ the $i$ th eigenvalue and call a function satisfying $\Delta \varphi+\mu_{i}(M) \varphi$ $=0$ an $i$ th eigenfunction.

\section{Main theorem.}

THEOREM 1. Suppose that $M$ is homeomorphic to $S^{2}$ and $\varphi_{1}, \varphi_{2}, \varphi_{3}$, are three first eigenfunctions such that their square sum is a constant. Then $M$ is actually isometric to a sphere with constant sectional curvature.

Proof. The assumption of the theorem says that

$$
\begin{cases}\Delta \varphi_{i}+\mu_{1}(M) \varphi_{i}=0, & i=1,2,3, \\ \sum_{i} \varphi_{i}^{2}=c, & c \text { is a constant. }\end{cases}
$$

Thus,

$$
\begin{aligned}
0 & =\Delta\left(\sum_{i} \varphi_{i}^{2}\right)=\sum_{i} 2\left|d \varphi_{i}\right|^{2}+2 \sum_{i} \varphi_{i} \Delta \varphi_{i} \\
& =\sum_{i} 2\left|d \varphi_{i}\right|^{2}-2 \mu_{1}(M) \sum_{i} \varphi_{i}^{2} .
\end{aligned}
$$

Then we have $\Sigma_{i}\left|d \varphi_{i}\right|^{2}=c \mu_{1}(M)$. Again we take Laplacian of both sides of

Received by the editors August 26, 1974 and, in revised form, March 26, 1975.

AMS (MOS) subject classifications (1970). Primary 53C99.

Key words and phrases. Eigenfunctions, eigenvalues. 
the above identity and obtain $\sum_{i} \Delta\left|d \varphi_{i}\right|^{2}=0$.

The Bochner-Lichnerowicz formula [1, p. 131] says that

$$
\frac{1}{2} \Delta|d f|^{2}=|\operatorname{Hess} f|^{2}+(d f, d \Delta f)+\operatorname{Ric}\left(d f^{\#}, d f^{\#}\right),
$$

where Ric denotes the Ricci curvature and $d f^{\#}$ is the vector field, naturally corresponds to $d f$. Therefore,

$$
\begin{aligned}
0 & =\sum_{i} \frac{1}{2} \Delta\left|d \varphi_{i}\right|^{2}=\sum_{i}\left|\operatorname{Hess} \varphi_{i}\right|^{2}+\sum_{i}\left(d \varphi_{i}, d \Delta \varphi_{i}\right)+\sum_{i} \operatorname{Ric}\left(d \varphi_{i}^{\#}, d \varphi_{i}^{\#}\right) \\
& =\sum_{i}\left|\operatorname{Hess} \varphi_{i}\right|^{2}-\mu_{1}(M)^{2} \sum_{i} \varphi_{i}^{2}+\sum_{i} \operatorname{Ric}\left(d \varphi_{i}^{\#}, d \varphi_{i}^{\#}\right) .
\end{aligned}
$$

Observe that 2-dim manifolds are Einstein manifolds. Hence

$$
\sum_{i} \operatorname{Ric}\left(d \varphi_{i}^{\#}, d \varphi_{i}^{\#}\right)=\tau \sum_{i}\left|d \varphi_{i}\right|^{2}
$$

where $\tau$ denotes the sectional curvature function. Consequently,

$$
\begin{aligned}
0 & =\sum_{i}\left|\operatorname{Hess} \varphi_{i}\right|^{2}-c \mu_{1}(M)^{2}+\tau c \mu_{1}(M) \\
& \geqslant \sum_{i} \frac{\left|\Delta \varphi_{i}\right|^{2}}{2}-c \mu_{1}(M)^{2}+\tau c \mu_{1}(M)=-c \mu_{1}(M)^{2} / 2+\tau c \mu_{1}(M) .
\end{aligned}
$$

Thus we have

$$
\mu_{1}(M) \geqslant 2 \tau .
$$

Integrating $(*)$ and using the Gauss-Bonnet formula, we have

$$
\mu_{1}(M) \times \operatorname{area}(M) \geqslant 8 \pi
$$

where area $(M)$ denotes the area of $M$.

Now we use a result of $\mathbf{J}$. Hersch [3] to complete the proof. His theorem says that if $M$ is homeomorphic to $S^{2}$, then $\mu_{1}(M) \leqslant 8 \pi / \operatorname{area}(M)$ and equality holds iff $M$ is indeed isometric to a sphere with constant sectional curvature.

2. Remarks. The proof of the theorem does not use the assumption that there are exactly three 1st eigenfunctions with square sum equal to one. Therefore, we have the following corollaries:

Corollary 2. Suppose that $(M, g)$ is a 2-dim $C^{\infty}$ compact Riemannian manifold and there exist eigenfunctions $\varphi_{1}, \ldots, \varphi_{m}$ such that $\Delta \varphi_{i}+\mu \varphi_{i}=0$ and $\sum_{i} \varphi_{i}^{2}$ equals to a constant. Then

$$
\mu \geqslant 2 \tau \text { and } \mu \geqslant 4 \pi \chi(M) / \operatorname{area}(M)
$$

where $\tau$ denotes the sectional curvature function of $M$.

COROLlary 3. Suppose that $(M, g)$ is a $2-\operatorname{dim} C^{\infty}$ compact minimally immersed submanifold of $S^{m}$ with the induced metric. Then, area $(M)$ $\geqslant 2 \pi \chi(M)$ and the sectional curvature is bounded above by 1 .

In fact, in Theorem $1, m=3$ is the only number such that there are $m$ 1st eigenfunctions with square sum equal to a constant. Since every nonconstant eigenfunction must vanish somewhere, this rules out the case $m=1 . m=2$ is 
not possible because by the results of [2] we have $\varphi_{1}^{-1}(0) \cap \varphi_{2}^{-1}(0) \neq \varnothing$ whenever $\Delta \varphi_{i}+\mu \varphi_{i}=0, i=1,2, \mu>0$. Also the results of [2] show that if $M$ is homeomorphic to $S^{2}$, then the multiplicity of the 1st eigenfunction is at most 3. This shows that $m>3$ is not possible.

\section{REFERENCES}

1. M. Berger, P. Gauduchon and E. Mazet, Le spectre d'une variété riemannienne, Lecture Notes in Math., vol. 194, Springer-Verlag, Berlin and New York, 1971. MR 43 \#8025.

2. S. Y. Cheng, Nodal sets and eigenfunctions, Comment. Math. Helv. (to appear).

3. J. Hersch, Quatre propriétés isopérimétriques de membranes sphériques homogènes, $\mathrm{C}$. R. Acad. Sci. Paris Sér. A-B 270 (1970), Al645-A1648. MR 45 \# 1444.

Department of Mathematics, University of California, Berkeley, California 94720

Current address: Courant Institute of Mathematical Sciences, New York University, New York, New York 10012 